"Baseball's All-Stars: Birthplace and Distribution"

by

Paul M. Sommers

June, 2002

MIDDLEBURY COLLEGE ECONOMICS DISCUSSION PAPER NO. 02-14

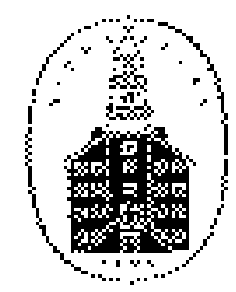

DEPARTMENT OF ECONOMICS

MIDDLEBURY COLLEGE

MIDDLEBURY, VERMONT 05753

http://www.middlebury.edu/ econ 


\section{Baseball's All-Stars: Birthplace and Distribution}

by

Paul M. Sommers

Department of Economics

Middlebury College

Middlebury, Vermont 05753

psommers@middlebury.edu 


\title{
Baseball's All-Stars: Birthplace and Distribution
}

\author{
Paul M. Sommers
}

\author{
You're not a star until they can \\ spell your name in Karachi. \\ — Humphrey Bogart
}

\section{Introduction}

In this brief note, we examine where All-Star baseball players were born and what (if any) regional patterns have emerged between 1933 when the midsummer classic began and 1999. With the exception of 1959-62, when players and owners decided to play two All-Star games instead of one, and 1945, when no game was played due to wartime travel restrictions, the interleague encounter has been an annual event. The number of players picked for each league's All-Star squad increased from 18 players in 1933 to its present 28 [2, p. 5]. In some years, however, the number of All-Stars has exceeded 28 because some ballplayers were named to their league's team but did not play due to injury. All-Star Game rosters are from the Web site www.baseball-almanac.com and birthplace information may be gleaned from The Baseball Encyclopedia [1] and Major League Baseball's Web site www.mlb.com.

\section{The Data}

Over the 67-year period (1933-99) 70 All-Star games were played with performers from all 50 states (but Nevada) and 18 foreign countries. Foreign born All-Stars since 1933 have come from Australia (one player appearance), Canada (17 player appearances), Canal Zone (18), Colombia (2), Cuba (74), Dominican Republic (83), 
England (4), France (1), Germany (1), Jamaica (6), Japan (1), Mexico (16), The Netherlands (2), Nicaragua (4), Panama (10), Puerto Rico (100), Scotland (3), and Venezuela (49).

All-Star games have included repeat performers (Hank Aaron was picked for 25 squads, Willie Mays and Stan Musial were picked for 24 each; Babe Ruth appeared in only the first two All-Star games), players who performed for both leagues (Johnny Mize, Frank Robinson, and Rich "Goose" Gossage, among others), and beginning in 1936 the first foreign born player (George Selkirk from Huntsville, Ontario, Canada). The Hank Aaron of 1955 is not the same ballplayer as the Hank Aaron of 1975. Hence, ballplayers who repeated as All-Stars are counted more than once.

Table 1 shows the distribution of players by birthplace across seven decades. In the 1990s, there were more All-Star selections from the state of California (25.6 percent of the U.S. total) than from the states of Florida, New York, Pennsylvania, and Ohio combined. Almost one-fifth (19.7 percent) of all All-Stars in the 1990s were foreign born. Of these foreign born ballplayers, almost two-thirds were from Puerto Rico (44) or the Dominican Republic (33). The first Latino All-Stars were Chico Carrasquel (Venezuela), Connie Marrero (Cuba), and Minnie Minoso (Cuba), all on the 1951 American League squad.

The numbers in Table 1 are adjusted for the size of each state's population (at the beginning of each decade) and the resulting rates (All-Stars per 100,000 population) are reported in Table 2. Census population figures at the beginning of each decade for each state are from The World Almanac and Book of Facts 1998 [3, pp. 380-81]. Interested readers could use the number of foreign born All-Stars from Puerto Rico, the Dominican Republic, and Cuba reported in Table 1, look up their populations, and calculate their rates of All-Star production. In the 1990s, for example, the rates of All-Star production from Puerto Rico (1.25 using a beginning-of-decade population of 3.52 million) and the Dominican Republic (0.39 using a population of 8.23 million) were well above the U.S. average, underscoring baseball's discovery of "new" talent in Latin American countries.

The five states with the highest rates (averaged across seven decades) are Alabama (.71), Arkansas (.68), Oklahoma (.64), California (.61), and Missouri (.51). Birdie Tebbetts alone accounts for Vermont's high rate of participation in the 1940s. Harmon 
Killebrew, Larry Jackson, and Vern Law account for Idaho's extraordinary rate of participation in the 1960s. When these seven rates (one for each decade for each state) are correlated with the midpoint of each decade, a positive correlation coefficient (above $.75)$ emerges for only one state, Florida $(r=.79)$. The correlation coefficient is negative (and less than -.75$)$ for the states of Iowa $(r=-.77)$, New Jersey $(r=-.83)$, North Carolina $(r=-.90)$, and Tennessee $(r=-.78)$.

\section{Chi-square Tests}

All fifty states and the District of Columbia were divided into four Census Regions as follows: Northeast (CT, ME, MA, NH, NJ, NY, PA, RI, VT); Midwest (IL, IN, IA, KS, MI, MN, MO, NE, ND, OH, SD, WI); West (AK, AZ, CA, CO, HI, ID, MT, NV, NM, OR, UT, WA, WY); and South (AL, AR, DE, DC, FL, GA, KY, LA, MD, MS, NC, OK, SC, TN, TX, VA, WV). A contingency table relating the four Census regions to the seven decades revealed that there were disproportionately more western born All-Stars in the 1980s and '90s ( $\chi^{2}=117.3, p<.0001$ ). Similar contingency tables (four rows, one for each Census region, and seven columns, one for each decade) were constructed for: American League (AL) All-Star pitchers (disproportionately high numbers from the West in the 1980s and '90s, $\chi^{2}=63.8, p<.0001$ ); AL All-Star hitters (disproportionately high numbers from the West in the 1940s and from the Midwest in the 1980s, $\chi^{2}=37.3, p=$ .005); National League (NL) All-Star pitchers (disproportionately many from the South in the 1940s and few from the West in the 1930s, $\chi^{2}=72.1, p<.0001$ ); and NL All-Star hitters (disproportionately many from the West in the 1980s and '90s, $\chi^{2}=147.7, p<$ .0001). Table 3 summarizes the regional results for pitchers/non-pitchers in the AL/NL.

Why are the results what they are, with disproportionately many western born AllStars in the 1980s and '90s? There are exceedingly many variables affecting the development of baseball's All-Stars, including the flourishing Little League baseball movement, the role played by college and even high school baseball programs, an expanding number of minor league teams of various classes, and even cultural differences. Franchise relocation and major league expansion might also have had an important effect on the development of western-born All-Stars. In the late 1950s, the NL 
Dodgers and Giants moved to the West Coast cities of Los Angeles and San Francisco. In 1961, the American League expanded to include the Los Angeles Angels who later moved to nearby Anaheim. The AL's Kansas City Athletics moved to Oakland, California in 1968. Major league baseball added two more West Coast teams (in San Diego and Seattle) in 1969 when each league was subdivided into six-team Eastern and Western divisions. Throughout the 1950s and '60s, western states attracted disproportionately many other migrants whose offspring flourished in an environment that promotes year-round outdoor activities. Perhaps not surprisingly, a generation later (in the 1980s and '90s) the West accounted for many of baseball's All-Stars.

Table 4 relates an All-Star player's birthplace to his league affiliation by decade. Disproportionately more foreign-born All-Stars represented the AL in the 1950s $\left(\chi^{2}=\right.$ 24.0, $p=.0001)$. Twenty-one of the 25 foreign born American League All-Stars were hitters, only four were pitchers. For each decade, similar contingency tables compared AL pitchers (hitters) with NL pitchers (hitters). By the 1960s, the NL foreign-born AllStars slightly exceeded those from the AL (55 v. 40). In the 1960s, the notable differences between leagues was the dominance of NL All-Star hitters and AL All-Star pitchers from the South $\left(\chi^{2}=29.0, p<.0001\right.$ for league differences between hitters; $\chi^{2}=13.1, p=.01$ for league differences between pitchers). In the 1970s, there was a dearth of NL All-Stars (especially hitters) from the Northeast $\left(\chi^{2}=10.4, p=.03\right.$ for league differences between hitters). The 1980s and '90s revealed disproportionately large numbers of NL hitters from the West $\left(\chi^{2}=32.8, p<.0001\right.$ in the 1980 s and $\chi^{2}=$ $25.2, p<.0001$ in the $1990 \mathrm{~s})$.

\section{Conclusion}

Since 1933 increasing numbers of U.S. born All-Star players have been Floridians or natives of western states, notably California. One cannot underestimate the allure of good weather and the arrival of not just one but six West Coast teams beginning in 1958. The most notable change over time, however, is the rising proportion of foreign born AllStars, with the vast majority of those players coming from Puerto Rico, the Dominican Republic, Cuba, and Venezuela. By the 1950s, the talent scarcity stateside prompted 
team owners to recruit outside the United States, notably in Latin America and the Caribbean. Of 392 total foreign born player appearances in All-Star games since 1933, close to 30 percent occurred in the 1990s. Thirty percent of the All-Stars who appeared in the 1999 All-Star Game alone were foreign born. Baseball's midsummer classic, which annually features the best talent from both leagues, has clearly become a "world" series. Viva baseball's All-Stars! 
Table 1. All-Stars by State of Birth and by Decade of Appearance, 1930s through 1990s

1930s 1940s 1950s 1960s 1970s 1980s 1990s Total

State

\begin{tabular}{|c|c|c|c|c|c|c|c|c|}
\hline Alabama & 5 & 15 & 23 & 59 & 32 & 19 & 10 & 163 \\
\hline Alaska & 0 & 0 & 0 & 0 & 0 & 0 & 3 & 3 \\
\hline Arizona & 1 & 3 & 0 & 0 & 0 & 0 & 1 & 5 \\
\hline Arkansas & 15 & 10 & 26 & 19 & 19 & 1 & 0 & 90 \\
\hline California & 40 & 75 & 74 & 64 & 80 & 125 & 123 & 581 \\
\hline Colorado & 0 & 2 & 0 & 0 & 8 & 8 & 0 & 18 \\
\hline Connecticut & 0 & 1 & 3 & 5 & 1 & 1 & 10 & 21 \\
\hline Delaware & 0 & 0 & 0 & 2 & 1 & 0 & 0 & 3 \\
\hline District of Columbia & 0 & 0 & 0 & 7 & 4 & 0 & 0 & 11 \\
\hline Florida & 1 & 1 & 1 & 16 & 22 & 38 & 33 & 112 \\
\hline Georgia & 8 & 23 & 8 & 2 & 3 & 8 & 15 & 67 \\
\hline Hawaii & 0 & 0 & 0 & 0 & 0 & 4 & 0 & 4 \\
\hline Idaho & 0 & 0 & 4 & 14 & 2 & 2 & 0 & 22 \\
\hline Illinois & 12 & 29 & 35 & 14 & 20 & 33 & 18 & 161 \\
\hline Indiana & 9 & 10 & 11 & 7 & 4 & 13 & 7 & 61 \\
\hline Iowa & 3 & 4 & 2 & 4 & 2 & 2 & 0 & 17 \\
\hline Kansas & 0 & 2 & 0 & 0 & 3 & 2 & 3 & 10 \\
\hline Kentucky & 3 & 13 & 11 & 8 & 1 & 3 & 5 & 44 \\
\hline Louisiana & 15 & 17 & 1 & 7 & 18 & 11 & 20 & 89 \\
\hline Maine & 0 & 0 & 0 & 0 & 1 & 1 & 0 & 2 \\
\hline Maryland & 17 & 12 & 7 & 12 & 2 & 11 & 18 & 79 \\
\hline Massachusetts & 6 & 13 & 8 & 11 & 9 & 6 & 15 & 68 \\
\hline Michigan & 7 & 7 & 15 & 21 & 31 & 10 & 11 & 102 \\
\hline Minnesota & 0 & 0 & 5 & 10 & 3 & 20 & 8 & 46 \\
\hline Mississippi & 3 & 7 & 3 & 1 & 10 & 9 & 3 & 36 \\
\hline Missouri & 14 & 26 & 31 & 42 & 16 & 10 & 9 & 148 \\
\hline Montana & 0 & 0 & 0 & 1 & 2 & 0 & 0 & 3 \\
\hline Nebraska & 4 & 2 & 4 & 13 & 2 & 6 & 8 & 39 \\
\hline Nevada & 0 & 0 & 0 & 0 & 0 & 0 & 0 & 0 \\
\hline New Hampshire & 3 & 1 & 0 & 2 & 1 & 0 & 1 & 8 \\
\hline New Jersey & 15 & 21 & 8 & 8 & 5 & 0 & 8 & 65 \\
\hline New Mexico & 0 & 7 & 6 & 0 & 0 & 0 & 2 & 15 \\
\hline New York & 25 & 26 & 50 & 58 & 35 & 19 & 32 & 245 \\
\hline North Carolina & 17 & 20 & 19 & 24 & 13 & 1 & 5 & 99 \\
\hline North Dakota & 0 & 0 & 0 & 0 & 0 & 0 & 1 & 1 \\
\hline Ohio & 5 & 14 & 15 & 28 & 42 & 42 & 26 & 172 \\
\hline Oklahoma & 15 & 14 & 23 & 25 & 21 & 5 & 6 & 109 \\
\hline Oregon & 0 & 1 & 2 & 1 & 5 & 10 & 3 & 22 \\
\hline Pennsylvania & 10 & 37 & 58 & 41 & 36 & 30 & 30 & 242 \\
\hline Rhode Island & 6 & 0 & 2 & 2 & 2 & 2 & 0 & 14 \\
\hline South Carolina & 5 & 14 & 17 & 7 & 5 & 11 & 2 & 61 \\
\hline South Dakota & 0 & 0 & 0 & 0 & 0 & 1 & 0 & 1 \\
\hline Tennessee & 10 & 6 & 7 & 12 & 5 & 7 & 3 & 50 \\
\hline Texas & 21 & 11 & 35 & 44 & 40 & 21 & 18 & 190 \\
\hline Utah & 0 & 0 & 0 & 0 & 0 & 1 & 0 & 1 \\
\hline
\end{tabular}


Table 1. All-Stars by State of Birth and by Decade of Appearance, 1930s through 1990s (Continued)

\begin{tabular}{lcccccccc} 
& $1930 \mathrm{~s}$ & $1940 \mathrm{~s}$ & $1950 \mathrm{~s}$ & $1960 \mathrm{~s}$ & $1970 \mathrm{~s}$ & $1980 \mathrm{~s}$ & $1990 \mathrm{~s}$ & Total \\
\multicolumn{1}{l}{ State } & & & & & & & & \\
& 0 & 4 & 0 & 0 & 6 & 4 & 1 & 15 \\
Vermont & 1 & 8 & 6 & 9 & 3 & 2 & 4 & 33 \\
Virginia & 6 & 0 & 4 & 8 & 9 & 6 & 11 & 44 \\
Washington & 0 & 1 & 6 & 7 & 8 & 9 & 4 & 35 \\
West Virginia & 4 & 13 & 14 & 5 & 0 & 1 & 2 & 39 \\
Wisconsin & 0 & 0 & 0 & 1 & 0 & 0 & 1 & 2 \\
Wyoming & 306 & 470 & 544 & 621 & 532 & 515 & 480 & 3468 \\
& & & & & & & & \\
U.S. Total & 2 & 5 & 29 & 95 & 69 & 75 & 117 & 392 \\
Foreign born & & & & & & & & \\
& & & & & & & & \\
Selected countries & 0 & 0 & 7 & 30 & 10 & 9 & 44 & 100 \\
$\quad \begin{array}{l}\text { Puerto Rico } \\
\text { Dominican Republic }\end{array}$ & 0 & 0 & 0 & 16 & 8 & 27 & 32 & 83 \\
$\quad$ Cuba & 0 & 0 & 11 & 31 & 21 & 4 & 7 & 74 \\
& & & & & & & & \\
Grand Total & 308 & 475 & 573 & 716 & 601 & 590 & 597 & 3860
\end{tabular}


Table 2. All-Stars per 100,000 Population by State of Birth and by Decade of Appearance, 1930s through 1990s

1930s 1940s 1950s 1960s 1970s 1980s 1990s Avg.

State

\begin{tabular}{|c|c|c|c|c|c|c|c|}
\hline Alabama & .19 & .53 & .75 & 1.81 & .93 & .49 & .25 \\
\hline Alaska & 0 & 0 & 0 & 0 & 0 & 0 & .55 \\
\hline Arizona & .23 & .60 & 0 & 0 & 0 & 0 & .03 \\
\hline Arkansas & .81 & .51 & 1.36 & 1.06 & .99 & .04 & 0 \\
\hline California & .70 & 1.09 & .70 & .41 & .40 & .53 & .41 \\
\hline Colorado & 0 & .18 & 0 & 0 & .36 & .28 & 0 \\
\hline Connecticut & 0 & .06 & .15 & .20 & .03 & .03 & .30 \\
\hline Delaware & 0 & 0 & 0 & .45 & .18 & 0 & 0 \\
\hline District of Columbia & 0 & 0 & 0 & .92 & .53 & 0 & 0 \\
\hline Florida & .07 & .05 & .04 & .32 & .32 & .39 & .26 \\
\hline Georgia & .28 & .74 & .23 & .05 & .07 & .15 & .23 \\
\hline Hawaii & 0 & 0 & 0 & 0 & 0 & .41 & 0 \\
\hline Idaho & 0 & 0 & .68 & 2.10 & .28 & .21 & 0 \\
\hline Illinois & .16 & .37 & .40 & .14 & .18 & .29 & .16 \\
\hline Indiana & .28 & .29 & .28 & .15 & .08 & .24 & .13 \\
\hline Iowa & .12 & .16 & .08 & .15 & .07 & .07 & 0 \\
\hline Kansas & 0 & .11 & 0 & 0 & .13 & .08 & .12 \\
\hline Kentucky & .11 & .46 & .37 & .26 & .03 & .08 & .14 \\
\hline Louisiana & .71 & .72 & .04 & .21 & .49 & .26 & .47 \\
\hline Maine & 0 & 0 & 0 & 0 & .10 & .09 & 0 \\
\hline Maryland & 1.04 & .66 & .30 & .39 & .05 & .26 & .38 \\
\hline Massachusetts & .14 & .30 & .17 & .21 & .16 & .10 & .25 \\
\hline Michigan & .14 & .13 & .24 & .27 & .35 & .11 & .12 \\
\hline Minnesota & 0 & 0 & .17 & .29 & .08 & .49 & .18 \\
\hline Mississippi & .15 & .32 & .14 & .05 & .45 & .36 & .12 \\
\hline Missouri & .39 & .69 & .78 & .97 & .34 & .20 & .18 \\
\hline Montana & 0 & 0 & 0 & .15 & .29 & 0 & 0 \\
\hline Nebraska & .29 & .15 & .30 & .92 & .13 & .38 & .51 \\
\hline Nevada & 0 & 0 & 0 & 0 & 0 & 0 & 0 \\
\hline New Hampshire & .64 & .20 & 0 & .33 & .14 & 0 & .09 \\
\hline New Jersey & .37 & .50 & .17 & .13 & .07 & 0 & .10 \\
\hline New Mexico & 0 & 1.32 & .88 & 0 & 0 & 0 & .13 \\
\hline New York & .20 & .19 & .34 & .35 & .19 & .11 & .18 \\
\hline North Carolina & .54 & .56 & .47 & .53 & .26 & .02 & .08 \\
\hline North Dakota & 0 & 0 & 0 & 0 & 0 & 0 & .16 \\
\hline Ohio & .08 & .20 & .19 & .29 & .39 & .39 & .24 \\
\hline Oklahoma & .63 & .60 & 1.03 & 1.07 & .82 & .17 & .19 \\
\hline Oregon & 0 & .09 & .13 & .06 & .24 & .38 & .11 \\
\hline Pennsylvania & .10 & .37 & .55 & .36 & .31 & .25 & .25 \\
\hline Rhode Island & .87 & 0 & .25 & .23 & .21 & .21 & 0 \\
\hline South Carolina & .29 & .74 & .80 & .29 & .19 & .35 & .06 \\
\hline South Dakota & 0 & 0 & 0 & 0 & 0 & .15 & 0 \\
\hline Tennessee & .38 & .21 & .21 & .34 & .13 & .15 & .06 \\
\hline Texas & .36 & .17 & .45 & .46 & .36 & .15 & .11 \\
\hline Utah & 0 & 0 & 0 & 0 & 0 & .07 & 0 \\
\hline
\end{tabular}


Table 2. All-Stars per 100,000 Population by State of Birth and by Decade of Appearance, 1930s through 1990s

(Continued)

\begin{tabular}{lcccccccc}
\multicolumn{1}{l}{ State } & 1930s & $1940 \mathrm{~s}$ & $1950 \mathrm{~s}$ & $1960 \mathrm{~s}$ & $1970 \mathrm{~s}$ & $1980 \mathrm{~s}$ & $1990 \mathrm{~s}$ & Avg. \\
& & & & & & & & \\
Vermont & 0 & 1.11 & 0 & 0 & .13 & .78 & .18 & .31 \\
Virginia & .04 & .30 & .18 & .23 & .06 & .04 & .06 & .13 \\
Washington & .38 & 0 & .17 & .28 & .26 & .15 & .23 & .21 \\
West Virginia & 0 & .05 & .30 & .38 & .46 & .46 & .22 & .27 \\
Wisconsin & .14 & .41 & .41 & .13 & 0 & .02 & .04 & .16 \\
Wyoming & 0 & 0 & 0 & .30 & 0 & 0 & .22 & .07 \\
U.S. Total & .25 & .36 & .36 & .35 & .26 & .23 & .19 & .29
\end{tabular}


Table 3. Contingency Tables Relating Census Region of Birth and Decade of Appearance,

Pitchers and Non-Pitchers by League

\begin{tabular}{cccccccc}
\multicolumn{8}{c}{ AL All-Star Pitchers } \\
& $1930 s$ & $1940 s$ & $1950 s$ & $1960 s$ & $1970 s$ & $1980 s$ & $1990 s$ \\
Northeast & 3 & 14 & 21 & 26 & 23 & 8 & 13 \\
Midwest & 12 & 23 & 18 & 29 & 21 & 27 & 21 \\
West & 8 & 8 & 16 & 13 & 18 & 34 & 31 \\
South & 22 & 25 & 27 & 37 & 21 & 17 & 16
\end{tabular}

AL All-Star Non-Pitchers

1930s 1940s 1950s 1960s 1970s 1980s 1990s

$\begin{array}{clllllll}\text { Northeast } & 24 & 31 & 37 & 40 & 40 & 31 & 30 \\ \text { Midwest } & 15 & 29 & 45 & 62 & 41 & 63 & 39 \\ \text { West } & 21 & 45 & 39 & 35 & 28 & 32 & 25 \\ \text { South } & 49 & 54 & 58 & 83 & 80 & 55 & 49\end{array}$

NL All-Star Pitchers

1930s 1940s 1950s 1960s 1970s 1980s 1990s

$\begin{array}{cccccccc}\text { Northeast } & 8 & 16 & 33 & 31 & 16 & 14 & 24 \\ \text { Midwest } & 11 & 8 & 24 & 23 & 21 & 20 & 12 \\ \text { West } & 0 & 7 & 12 & 24 & 23 & 21 & 27 \\ \text { South } & 20 & 37 & 14 & 16 & 22 & 24 & 26\end{array}$

NL All-Star Non-Pitchers

1930s 1940s 1950s 1960s 1970s 1980s 1990s

$\begin{array}{cccccccc}\text { Northeast } & 30 & 42 & 38 & 30 & 17 & 13 & 30 \\ \text { Midwest } & 20 & 47 & 45 & 30 & 40 & 30 & 21 \\ \text { West } & 18 & 28 & 23 & 17 & 37 & 69 & 61 \\ \text { South } & 45 & 56 & 94 & 125 & 84 & 60 & 55\end{array}$




\section{Table 4. Contingency Tables Relating Census Region of Birth and Player's League Affiliation, by Decade}

\begin{tabular}{|c|c|c|c|c|c|}
\hline & & & & & \\
\hline & Northeast & Midwest & West & South & Foreign \\
\hline AL pitchers & 21 & 18 & 16 & 27 & 4 \\
\hline NL pitchers & 33 & 24 & 12 & 14 & 1 \\
\hline AL non-pitchers & 37 & 45 & 39 & 58 & 21 \\
\hline NL non-pitchers & 38 & 45 & 23 & 94 & 3 \\
\hline & & & & & \\
\hline & Northeast & Midwest & West & South & Foreign \\
\hline AL pitchers & 26 & 29 & 13 & 37 & 12 \\
\hline NL pitchers & 31 & 23 & 24 & 16 & 16 \\
\hline Al non-pitchers & 40 & 62 & 35 & 83 & 28 \\
\hline NL non-pitchers & 30 & 30 & 17 & 125 & 39 \\
\hline & & & & & \\
\hline & Northeast & Midwest & West & South & Foreign \\
\hline AL pitchers & 23 & 21 & 18 & 21 & 8 \\
\hline NL pitchers & 16 & 21 & 23 & 22 & 5 \\
\hline AL non-pitchers & 40 & 41 & 28 & 80 & 28 \\
\hline NL non-pitchers & 17 & 40 & 37 & 84 & 28 \\
\hline
\end{tabular}




\section{Table 4. Contingency Tables Relating Census Region of Birth and Player's League Affiliation, by Decade \\ (Continued)}

\begin{tabular}{|c|c|c|c|c|c|}
\hline & \multicolumn{4}{|c|}{$1980 s$} & \multirow[b]{2}{*}{ Foreign } \\
\hline & Northeast & Midwest & West & South & \\
\hline AL pitchers & 5 & 27 & 34 & 17 & 6 \\
\hline NL pitchers & 14 & 20 & 21 & 24 & 13 \\
\hline AL non-pitchers & 31 & 63 & 32 & 55 & 27 \\
\hline \multirow[t]{3}{*}{ NL non-pitchers } & 13 & 30 & 69 & 60 & 29 \\
\hline & \multicolumn{4}{|c|}{$1990 \mathrm{~s}$} & \\
\hline & Northeast & Midwest & West & South & Foreign \\
\hline AL pitchers & 13 & 21 & 31 & 16 & 16 \\
\hline NL pitchers & 24 & 12 & 27 & 26 & 11 \\
\hline AL non-pitchers & 30 & 39 & 25 & 49 & 55 \\
\hline NL non-pitchers & 30 & 21 & 61 & 55 & 35 \\
\hline
\end{tabular}




\section{References}

1. The Baseball Encyclopedia (eleventh edition), Macmillan Publishing Company, New York, 1999.

2. R. Obojski, All-Star Baseball Since 1933, Stein and Day, New York, 1980.

3. The World Almanac and Book of Facts 1998, World Almanac Books, Mahwah, New Jersey, 1997. 